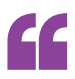

Intriguingly, these

functional and structural deficits occur before the onset of disease...

\title{
Mitochondrial dysfunction could precipitate motor neuron loss in spinal muscular atrophy
}

Mitochondrial dysfunction and subsequent perturbations in energy production could underlie damage to motor neurons in spinal muscular atrophy (SMA), according to a new mouse model study. The finding could help to explain why motor neurons are predominantly affected in SMA, and to eventually pave the way to mechanism-based therapies.

SMA is the most common genetic cause of infant death. No curative therapy exists for the disease, though a number of therapeutic strategies are currently being evaluated in clinical trials.

SMA is caused by a mutation in survival motor neuron 1 gene (SMN1), which leads to reduced levels of SMN protein. All cells are dependent on functional SMN; however, insufficient SMN levels seem to predominantly affect tissues and cells that have high energy demand, such as motor neurons, skeletal muscles and the heart. Even though the genetic cause for SMA is known, the mechanisms of this tissue-specific vulnerability are poorly understood, which has hampered development of clinically effective therapies.

Yong-Chao Ma and colleagues from the Northwestern University Feinberg School of Medicine analysed the transcriptome of spinal motor neurons in a transgenic SMA mouse model that shows molecular and pathological characteristics mimicking those seen in human patients. "Unexpectedly, we found altered expression of several genes associated with mitochondrial energy production," says Ma.

The changes in gene expression were reflected in several other measures that indicated structural and functional deficits in motor neuron mitochondria in SMA, such as increased oxidative stress and reduced mitochondrial respiration and retrograde transport. Moreover, the researchers observed notable mitochondrial fragmentation and swelling.

"Intriguingly, these functional and structural deficits occur before the onset of disease symptoms, suggesting a role for mitochondrial dysfunction in initiation of SMA pathogenesis, rather than being a secondary effect of motor neuron degeneration," Ma comments.

Defective mitochondrial bioenergetics could explain why SMA predominantly affects fast fatigable motor neurons and other tissues with high metabolic rates. Moreover, motor neurons - which have very long axons - could be particularly vulnerable to impaired transport of mitochondria.

According to $\mathrm{Ma}$, the newly discovered critical role for mitochondrial defects in SMA pathogenesis could be a novel therapeutic target that could complement current therapeutic strategies that aim to increase SMN production.

Hemi Malkki

ORIGINAL ARTICLE Miller, N. et al. Motor neuron mitochondrial dysfunction in spinal muscular atrophy. Hum. Mol.Genet. http://dx.doi.org/ 10.1093/hmg/ddw262 (2016)

FURTHER READING Faravelli, I. et al. Spinal muscular atrophy - recent therapeutic advances for an old challenge. Nat. Rev. Neurol. 11, 351-359 (2015) 